\title{
Lasry-Lions Regularization and a Lemma of Ilmanen
}

\author{
PAtrick Bernard (*)
}

Let $H$ be a Hilbert space. We define the following inf (sup) convolution operators acting on bounded functions $u: H \longrightarrow \mathbb{R}$ :

$$
T_{t} u(x):=\inf _{y}\left(u(y)+\frac{1}{t}\|y-x\|^{2}\right)
$$

and

$$
\check{T}_{t} u(x):=\sup _{y}\left(u(y)-\frac{1}{t}\|y-x\|^{2}\right) .
$$

We have the relation

$$
T_{t}(-u)=-\check{T}_{t}(u)
$$

Recall that these operators form semi-groups, in the sense that

$$
T_{t} \circ T_{s}=T_{t+s} \quad \text { and } \quad \check{T}_{t} \circ \check{T}_{s}=\check{T}_{t+s}
$$

for all $t \geqslant 0$ and $s \geqslant 0$, as can be checked by direct calculation. Note also that

$$
\inf u \leqslant T_{t} u(x) \leqslant u(x) \leqslant \check{T}_{t} u(x) \leqslant \sup u
$$

for each $t \geqslant 0$ and each $x \in H$. A function $u: H \longrightarrow \mathbb{R}$ is called $k$-semiconcave, $k>0$, if the function $x \longrightarrow u(x)-\|x\|^{2} / k$ is concave. We will occasionally consider semi-concave functions which take values in $[-\infty,+\infty)$. The function $u$ is called $k$-semi-convex if $-u$ is $k$-semi-concave. A function $u$ is $t$-semi-concave and upper semi-continous if and only if it belongs to the image of the operator $T_{t}$, this follows from Lemma 1 and Lemma 3 below. A function is called semi-concave if it is $k$-semi-concave for some $k>0$. A function $u$ is said $C^{1,1}$ if it is Frechet differentiable and if the gradient of $u$ is Lipschitz. Note that a continuous function $u: H \longrightarrow \mathbb{R}$ is $C^{1,1}$

(*) Indirizzo dell'A.: CEREMADE, Université Paris-Dauphine, Place du Maréchale de Lattre de Tassigny, 75775, Paris Cedex 16, France.

E-mail: patrick.bernard@ceremade.dauphine.fr 
if and only if it is semi-concave and semi-convex, see Lemma 5. Let us recall two important results in that language:

Theorem 1 (Lasry-Lions, [6]). Let $u$ be a bounded function. For $0<s<t$, the function $\breve{T}_{s} \circ T_{t} u$ is $C^{1,1}$ and, if $u$ is uniformly continuous, then it converges uniformly to $u$ when $t \longrightarrow 0$.

THEOREM 2 (Ilmanen, [5]). Let $u \geqslant v$ be two bounded functions on $H$ such that $u$ and $-v$ are semi-concave. Then there exists a $C^{1,1}$ function $w$ such that $u \geqslant w \geqslant v$.

Our goal in the present paper is to "generalize" simultaneously both of these results as follows:

THeorem 3. The operator $R_{t}:=\check{T}_{t} \circ T_{2 t} \circ \check{T}_{t}$ has the following properties:

- Regularization : For each function $f: H \longrightarrow \mathbb{R}$ and each $t>0$, the function $R_{t}(f)$ is $C^{1,1}$ provided it is locally bounded. This holds for all $t>0$ if $f$ is bounded.

- Approximation : If $f: H \longrightarrow \mathbb{R}$ is uniformly continuous, then $R_{t}(f)$ is $C^{1,1}$ and converges uniformly to $f$ as $t \longrightarrow 0$.

- Pinching: If there exists a k-semi-concave continuous function u and a $k$-semi-convex continuous function $v$ such that $v \leqslant f \leqslant u$, then, for all $t \in] 0, k]$, we have $u \geqslant R_{t}(f) \geqslant v$, and $R_{t}(f)$ is $C^{1,1}$.

Theorem 3 does not, properly speaking, generalize Theorem 5. However, it offers a new (although similar) answer to the same problem: approximating uniformly continuous functions on Hilbert spaces by $C^{1,1}$ functions with a simple explicit formula.

Because of its symmetric form, the regularizing operator $R_{t}$ enjoys some nicer properties than the Lasry-Lions operators. For example, if $f$ is $C^{1,1}$, then it follows from the pinching property that $R_{t} f=f$ for $t$ small enough.

Theorem 2, can be proved using Theorem 3 by taking $w=R_{t} u$, for $t$ small enough. Note, in view of Lemma 3 bellow, that $R_{t} u=\check{T}_{t} \circ T_{t} u$ when $t$ is small enough.

Theorem 3 can be somehow extended to the case of finite dimensional open sets or manifolds via partition of unity, at the price of loosing the simplicity of explicit expressions. Let $M$ be a paracompact manifold of dimension $n$, equipped once and for all with an atlas $\left(\phi_{i}, i \in \Im\right)$ composed of 
charts $\phi_{i}: B^{n} \longrightarrow M$, where $B^{n}$ is the open unit ball of radius one centered at the origin in $\mathbb{R}^{n}$. We assume in addition that the image $\phi_{i}\left(B^{n}\right)$ is a relatively compact open set, and that the sets $\phi_{i}\left(B^{n}\right), i \in \mathfrak{s}$ form a locally finite open covering of the manifold $M$. Let us fix, once and for all, a

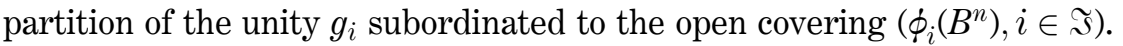
It means that the function $g_{i}$ is non-negative with support inside $\phi_{i}\left(B^{n}\right)$, and that $\sum_{i} g_{i}=1$ (note that this sum is finite at each point). Let us define the operator

$$
G_{t}(u):=\sum_{i}\left[R_{t a_{i}}\left(\left(g_{i} u\right) \circ \phi_{i}\right)\right] \circ \phi_{i}^{-1},
$$

where $a_{i}, i \in \Im$ are positive real numbers. In this expression, we consider each of the terms $\left.\left[R_{t a_{i}}\left(g_{i} u\right) \circ \phi_{i}\right)\right] \circ \phi_{i}^{-1}$ as defined on the whole manifold $M$ with the value 0 outside of $\phi_{i}\left(B^{n}\right)$. The sum is then locally finite hence well-defined. We say that a function $u: M \longrightarrow \mathbb{R}$ is locally semi-concave if, for each $i \in \mathfrak{I}$, there exists a constant $b_{i}$ such that the function $u \circ \phi_{i}-\|\cdot\|^{2} / b_{i}$ is concave on $B^{n}$.

THEOREM 4. Let $u \geqslant v$ be two continuous functions on $M$ such that $u$ and $-v$ are locally semi-concave. Then, the real numbers $a_{i}$ can be chosen such that, for each $t \in] 0,1]$ and each function $f$ satisfying $u \geqslant f \geqslant v$, we have:

- The function $G_{t}(f)$ is locally $C^{1,1}$.

- If $f$ is continuous, then $G_{t}(f)$ converges locally uniformly to $f$ as $t \longrightarrow 0$.

$$
\text { - } u \geqslant G_{t}(f) \geqslant v \text {. }
$$

We will give some properties, most of which are well-known, of the operators $T_{t}$ and $\check{T}_{t}$ in Section 1, and derive the proof of the main results in Section 2.

Notes and Acknowledgements. Theorem 2 appears in Ilmanen's paper [5] as Lemma 4G. Several proofs are sketch there but none is detailed. The proof we detail here follows lines similar to one of the sketches of Ilmanen. This statement also has a more geometric counterpart, Lemma 4E in [5]. A detailed proof of this geometric version is given in [2], Appendix. My attention was attracted to these statements and their relations with recent progresses on sub-solutions of the Hamilton-Jacobi equation (see [4, 1, 7]) by Pierre Cardialaguet, Albert Fathi and Maxime Zavidovique. Albert Fathi and Maxime Zavidovique also recently wrote a detailed proof of 
Theorem 1, see [3]. This paper also proves how the geometric version follows from Theorem 2. There are many similarities between the tools used in the present paper and those used in [1]. Moreover, Maxime Zavidovique observed in [7] that the existence of $C^{1,1}$ subsolutions of the Hamilton-Jacobi equation in the discrete case can be deduced from Theorem 2. However, is seems that the main result of [1] (the existence of $C^{1,1}$ subsolutions in the continuous case) can't be deduced easily from Theorem 2. Neither can Theorem 2 be deduced from it.

\section{The operators $T_{t}$ and $\check{T}_{t}$ on Hilbert spaces.}

The proofs of the theorems follow from standard properties of the operators $T_{t}$ and $\check{T}_{t}$ that we now recall in details.

Lemma 1. For each function $u: H \longrightarrow \mathbb{R}$, the function $T_{t} u$ (which takes values in $[-\infty,+\infty)$ ), is $t$-semi-concave and upper semi-continous. The function $\check{T}_{t} u$ (which takes values in $(-\infty,+\infty]$ ), is $t$-semi-convex and lower semi-continuous. Moreover, if $u$ is $k$-semi-concave, then for each $t<k$ the function $\check{T}_{t} u$ is $(k-t)$-semi-concave. Similarly, if $u$ is $k$-semi-convex, then for each $t<k$ the function $T_{t} u$ is $(k-t)$-semi-convex.

Proof. We shall prove the statements concerning $T_{t}$. We have

$$
\begin{aligned}
T_{t} u(x)-\|x\|^{2} / t=\inf _{y}\left(u(y)+\|y-x\|^{2} / t-\|x\|^{2} / t\right)= & \\
& =\inf _{y}\left(u(y)+\|y\|^{2} / t-2 x \cdot y / t\right),
\end{aligned}
$$

this function is concave and upper semi-continuous as an infimum of continuous linear functions. On the other hand, we have

$$
T_{t} u(x)+\|x\|^{2} / l=\inf _{y}\left(u(y)+\|y-x\|^{2} / t+\|x\|^{2} / l\right) .
$$

Setting $f(x, y):=u(y)+\|y-x\|^{2} / t+\|x\|^{2} / l$, the function $\inf _{y} f(x, y)$ is a convex function of $x$ if $f$ is a convex function of $(x, y)$. This is true if $u$ is $k$ semi-convex, $t<k$, and $l=k-t$ because we have the expression

$$
f(x, y)=u(y)+\|y-x\|^{2} / t+\|x\|^{2} / l=\left(u(y)+\|y\|^{2} / k\right)+\left\|\sqrt{\frac{l}{k t}} y-\sqrt{\frac{k}{l t}} x\right\|^{2} .
$$


Given a uniformly continuous function $u: H \longrightarrow \mathbb{R}$, we define its modulus of continuity $\rho(r):[0, \infty) \longrightarrow[0, \infty)$ by the expression $\rho(r)=$ $\sup _{x, e} u(x+r e)-u(x)$, where the supremum is taken on all $x \in H$ and all $e$ in the unit ball of $H$. The function $\rho$ is non-decreasing, it satisfies $\rho\left(r+r^{\prime}\right) \leqslant \rho(r)+\rho\left(r^{\prime}\right)$, and it converges to zero in zero (this last fact is equivalent to the uniform continuity of $u$ ). We say that a function $\rho:[0, \infty) \longrightarrow[0, \infty)$ is a modulus of continuity if it satisfies these properties. Given a modulus of continuity $\rho(r)$, we say that a function $u$ is $\rho$ continuous if $|u(y)-u(x)| \leqslant \rho(\|y-x\|)$ for all $x$ and $y$ in $H$.

LEMma 2. If $u: H \longrightarrow \mathbb{R}$ is uniformly continuous, then the functions $T_{t} u$ and $\check{T}_{t} u$ converge uniformly to $u$ when $t \longrightarrow 0$. Moreover, given a modulus of continuity $\rho$, there exists a non-decreasing function $\varepsilon(t):[0, \infty) \longrightarrow[0, \infty)$ satisfying $\lim _{t \rightarrow 0} \varepsilon(t)=0$ and such that, for each $\rho$ continuous bounded function $u$, we have:

- $T_{t} u$ and $\check{T}_{t} u$ are $\rho$-continuous for each $t \geqslant 0$.

- $u-\varepsilon(t) \leqslant T_{t} u(x) \leqslant u$ and $u \leqslant \check{T}_{t} u \leqslant u+\varepsilon(t)$ for each $t \geqslant 0$.

Proof. Let us fix $y \in H$, and set $v(x)=u(x+y)$. We have $u(x)-\rho(\|y\|) \leqslant v(x) \leqslant u(x)+\rho(\|y\|)$. Applying the operator $T_{t}$ gives $T_{t} u(x)-\rho(y) \leqslant T_{t} v(x) \leqslant T_{t} u(x)+\rho(y)$. On the other hand, we have

$$
\begin{array}{r}
\left.T_{t} v(x)=\inf _{z}(u(z+y)+\| z-x) \|^{2} / t\right)=\inf _{z}\left(u(z)+\|z-(x+y)\|^{2} / t\right)= \\
T_{t} u(x+y),
\end{array}
$$

so that

$$
T_{t} u(x)-\rho(\|y\|) \leqslant T_{t} u(x+y) \leqslant T_{t} u(x)+\rho(\|y\|) .
$$

We have proved that $T_{t} u$ is $\rho$ continuous if $u$ is, the proof for $\check{T}_{t} u$ is the same. have

In order to study the convergence, let us set $\varepsilon(t)=\sup _{r>0}\left(\rho(r)-r^{2} / t\right)$. We

$$
\varepsilon(t)=\sup _{r>0}\left(\rho(r \sqrt{t})-r^{2}\right) \leqslant \sup _{r>0}\left((r+1) \rho(\sqrt{t})-r^{2}\right)=\rho(\sqrt{t})+\rho^{2}(\sqrt{t}) / 4 .
$$

We conclude that $\lim _{t \rightarrow 0} \varepsilon(t)=0$. We now come back to the operator $T_{t}$, and observe that

$$
u(y)-\|y-x\|^{2} / t \geqslant u(x)-\rho(\|y-x\|)+\|y-x\|^{2} / t \geqslant u(x)-\varepsilon(t)
$$

for each $x$ and $y$, so that

$$
u-\varepsilon(t) \leqslant T_{t} u \leqslant u .
$$


LEMMA 3. For each function $u: H \longrightarrow(-\infty,+\infty]$, we have $\check{T}_{t} \circ T_{t}(u) \leqslant u$ and the equality $\check{T}_{t} \circ T_{t}(u)=u$ holds if and only if $u$ is $t$-semi-convex and lower semi-continuous. Similarly, given a function $v: H \longrightarrow[-\infty,+\infty)$, we have $T_{t} \circ \check{T}_{t}(v) \geqslant v$, with equality if and only if $v$ is $t$-semi-concave and upper semi-continuous.

Proof. Let us write explicitly

$$
\check{T}_{t} \circ T_{t} u(x)=\sup _{y} \inf _{z}\left(u(z)+\|z-y\|^{2} / t-\|y-x\|^{2} / t\right) .
$$

Taking $z=x$, we obtain the estimate $\check{T}_{t} \circ T_{t} u(x) \leqslant \sup _{y} u(z)=u(z)$. Let us now write

$$
\check{T}_{t} \circ T_{t} u(x)+\|x\|^{2} / t=\sup _{y} \inf _{z}\left(u(z)+\|z\|^{2} / t+(2 y / t) \cdot(x-z)\right)
$$

which by an obvious change of variable leads to

$$
\check{T}_{t} \circ T_{t} u(x)+\|x\|^{2} / t=\sup _{y} \inf _{z}\left(u(z)+\|z\|^{2} / t+y \cdot(x-z)\right) .
$$

We recognize here that the function $\check{T}_{t} \circ T_{t} u(x)+\|x\|^{2} / t$ is the Legendre bidual of the function $u(x)+\|x\|^{2} / t$. It is well-know that a function is equal to its Legendre bidual if and only if it is convex and lower semicontinuous.

\section{Proof of the main results.}

Proof of Theorem 3. For each function $f$ and each $t>0$, the function $\check{T}_{t} \circ T_{2 t} \circ \check{T}_{t} f$ is both $t$-semi-concave and $t$-semi-convex. It is $t$-semi-convex by Lemma 1 , and it is semi-concave because $T_{2 t}\left(\breve{T}_{t} f\right)$ is $2 t$-semi-concave by Lemma 1 , which implies, still by Lemma 1 , that $\check{T}_{t} \circ T_{2 t} \circ \check{T}_{t} f$ is $t$-semiconcave. As a consequence, Lemma 5 below implies that the function $R_{t} f$ is $C^{1,1}$ provided it is locally bounded. The function $R_{t}(f)$ is bounded if $f$ is bounded, hence its is $C^{1,1}$ in this case.

In the case where $f$ is uniformly continuous, Lemma 2 implies that

$$
f-\varepsilon(2 t) \leqslant R_{t}(f) \leqslant f+2 \varepsilon(t) .
$$

As a consequence, $R_{t}(f)$ is converging uniformly to $f$, and it is locally bounded hence $C^{1,1}$.

We now consider two continuous functions $u$ and $v$ such that $u$ and $-v$ are $k$ semi-concave, and such that $v \leqslant u$. We claim that

$$
u \geqslant f \geqslant v \Longrightarrow u \geqslant T_{t} \circ \check{T}_{t} f \geqslant v \text { and } u \geqslant \check{T}_{t} \circ T_{t} f \geqslant v
$$


for $t \leqslant k$. This claim implies that $u \geqslant \check{T}_{t} \circ T_{2 t} \circ \check{T}_{t} f \geqslant v$ when $u \geqslant f \geqslant v$ and $t \leqslant k$. Let us now prove the claim concerning $\check{T}_{t} \circ T_{t}$, the other part being similar. Since $v$ is $k$-semi-convex and continuous, we have $\check{T}_{t} \circ T_{t} v=v$ for $t \leqslant k$, by Lemma 3 . Then,

$$
u \geqslant f \geqslant \check{T}_{t} \circ T_{t} f \geqslant \check{T}_{t} \circ T_{t} v=v
$$

where the second inequality follows from Lemma 3, and the third from the obvious fact that the operators $T_{t}$ and $\check{T}_{t}$ are order-preserving.

We have proved that $v \leqslant R_{t}(f) \leqslant u$ if $v \leqslant f \leqslant u$ and $t \leqslant k$. For $\left.\left.t \in\right] 0, k\right]$, the function $R_{t}(f)$ is thus locally bounded hence $C^{1,1}$.

Proof of Theorem 4. Let $a_{i}$ be chosen such that the functions $\left(g_{i} u\right) \circ \phi_{i}$ and $-\left(g_{i} v\right) \circ \phi_{i}$ are $a_{i}$-semi-concave on $\mathbb{R}^{n}$ (when extended by 0 outside of $B^{n}$ ). The existence of real numbers $a_{i}$ with this property follows from Lemma 4 below. Given $u \geqslant f \geqslant v$, we can apply Theorem 3 for each $i$ to the functions

$$
\left(g_{i} u\right) \circ \phi_{i} \geqslant\left(g_{i} f\right) \circ \phi_{i} \geqslant\left(g_{i} v\right) \circ \phi_{i}
$$

extended by zero outside of $B^{n}$. We conclude that, for $\left.\left.t \in\right] 0,1\right]$, the function $R_{t a_{i}}\left(\left(g_{i} f\right) \circ \phi_{i}\right)$ is $C^{1,1}$ and satisfies

$$
\left(g_{i} u\right) \circ \phi_{i} \geqslant R_{t a_{i}}\left(\left(g_{i} f\right) \circ \phi_{i}\right) \geqslant\left(g_{i} v\right) \circ \phi_{i} .
$$

As a consequence, the function

$$
\left[R_{t a_{i}}\left(\left(g_{i} f\right) \circ \phi_{i}\right)\right] \circ \phi_{i}^{-1}
$$

extended as a function on $M$ equal to 0 outside of $\phi_{i}\left(B^{n}\right)$, is $C^{1,1}$. The function $G_{t}(f)$ is thus locally a finite sum of $C^{1,1}$ functions hence it is locally $C^{1,1}$. Moreover, we have

$$
u=\sum_{i} g_{i} u \geqslant G_{t}(f) \geqslant \sum_{i} g_{i} v=v
$$

We have used:

LEMma 4. Let $u: B^{n} \longrightarrow \mathbb{R}$ be a bounded function such that $u-\|\cdot\|^{2} / a$ is concave, for some $a>0$. For each compactly supported non-negative $C^{2}$ function $g: B^{n} \longrightarrow \mathbb{R}$, the product gu (extended by zero outside of $B^{n}$ ) is semi-concave on $\mathbb{R}^{n}$.

Proof. Since $u$ is bounded, we can assume that $u \geqslant 0$ on $B^{n}$. Let $K \subset B^{n}$ be a compact subset of the open ball $B^{n}$ which contains the support 
of $g$ in its interior. Since the function $u-\|\cdot\|^{2} / a$ is concave on $B_{1}$ it admits super-differentials at each point. As a consequence, for each $x \in B^{n}$, there exists a linear form $l_{x}$ such that

$$
0 \leqslant u(y) \leqslant u(x)+l_{x} \cdot(y-x)+\|y-x\|^{2} / a
$$

for each $y \in B^{1}$. Moreover, the linear form $l_{x}$ is bounded independently of $x \in K$. We also have

$$
0 \leqslant g(y) \leqslant g(x)+d g_{x} \cdot(y-x)+C\|y-x\|^{2}
$$

for some $C>0$, for all $x, y$ in $\mathbb{R}^{n}$. Taking the product, we get, for $x \in K$ and $y \in B^{n}$

$$
\begin{aligned}
& u(y) g(y) \leqslant u(x) g(x)+\left(g(x) l_{x}+u(x) d g_{x}\right) \cdot \\
& \cdot(y-x)+C\|y-x\|^{2}+C\|y-x\|^{3}+C\|y-x\|^{4}
\end{aligned}
$$

where $C>0$ is a constant independent of $x \in K$ and $y \in B^{n}$, which may change from line to line. As a consequence, setting $L_{x}=g(x) l_{x}+u(x) d g_{x}$, we obtain the inequality

$$
(g u)(y) \leqslant(g u)(x)+L_{x} \cdot(y-x)+C\|y-x\|^{2}
$$

for each $x \in K$ and $y \in B^{n}$. If we set $L_{x}=0$ for $x \in \mathbb{R}^{n}-K$, the relation (L) holds for each $x \in \mathbb{R}^{n}$ and $y \in \mathbb{R}^{n}$. For $x \in K$ and $y \in B^{n}$, we have already proved it. Since the linear forms $L_{x}, x \in K$ are uniformly bounded, we can assume that $L_{x} \cdot(y-x)+C\|y-x\|^{2} \geqslant 0$ for all $x \in K$ and $y \in \mathbb{R}^{n}-B^{n}$ by taking $C$ large enough. Then, (L) holds for all $x \in K$ and $y \in \mathbb{R}^{n}$. For $x \in \mathbb{R}^{n}-K$ and $y$ outside of the support $g$, the relation (L) holds in an obvious way, because $g u(x)=g u(y)=0$, and $L_{x}=0$. For $x \in \mathbb{R}^{n}-K$ and $y$ in the support of $g$, the relation holds provided that $C \geqslant \max (g u) / d^{2}$, where $d$ is the distance between the complement of $K$ and the support of $g$. This is a positive number since $K$ is a compact set containing the support of $g$ in its interior. We conclude that the function $(g u)$ is semi-concave on $\mathbb{R}^{n}$.

For completeness, we also prove, following Fathi:

Lemma 5. Let $u: H \longrightarrow \mathbb{R}$ be a locally bounded function which is both $k$-semi-concave and $k$-semi-convex. Then the function $u$ is $C^{1,1}$, and $6 / k$ is a Lipschitz constant for the gradient of $u$.

Proof. It is well known that a locally bounded convex function is continuous. We conclude that $u$ is continuous. Let $u$ be a continuous function which is both $k$-semi-concave and $k$-semi-convex. Then, for each $x \in H$, 
there exists a unique $l_{x} \in H$ such that

$$
\left|u(x+y)-u(x)-l_{x} \cdot y\right| \leqslant\|y\|^{2} / k .
$$

We conclude that $l_{x}$ is the gradient of $u$ at $x$, and we have to prove that the map $x \longmapsto l_{x}$ is Lipschitz. We have, for each $x, y$ and $z$ in $H$ :

$$
\begin{gathered}
l_{x} \cdot(y+z)-\|y+z\|^{2} / k \leqslant u(x+y+z)-u(x) \leqslant l_{x} \cdot(y+z)+\|y+z\|^{2} / k \\
l_{(x+y)} \cdot(-y)-\|y\|^{2} / k \leqslant u(x)-u(x+y) \leqslant l_{(x+y)} \cdot(-y)+\|y\|^{2} / k \\
l_{(x+y)} \cdot(-z)-\|z\|^{2} / k \leqslant u(x+y)-u(x+y+z) \leqslant l_{(x+y)} \cdot(-z)+\|z\|^{2} / k .
\end{gathered}
$$

Taking the sum, we obtain

$$
\left|\left(l_{x+y}-l_{x}\right) \cdot(y+z)\right| \leqslant\|y+z\|^{2} / k+\|y\|^{2} / k+\|z\|^{2} / k .
$$

By a change of variables, we get

$$
\left|\left(l_{x+y}-l_{x}\right) \cdot(z)\right| \leqslant\|z\|^{2} / k+\|y\|^{2} / k+\|z-y\|^{2} / k .
$$

Taking $\|z\|=\|y\|$, we obtain

$$
\left|\left(l_{x+y}-l_{x}\right) \cdot(z)\right| \leqslant 6\|z\|\|\| y \| / k
$$

for each $z$ such that $\|z\|=\|y\|$, we conclude that

$$
\left\|l_{x+y}-l_{x}\right\| \leqslant 6\|y\| / k \text {. }
$$

\section{REFERENCES}

[1] P. Bernard, Existence of $C^{1,1}$ critical sub-solutions of the Hamilton-Jacobi equation on compact manifolds, Ann. Sci. E. N. S. (4), 40, no. 3 (2007), pp. $445-452$.

[2] P. Cardaliaguet, Front Propagation Problems with Nonlocal Terms 2, J. Math. An. Appl., 260 (2001), pp. 572-601.

[3] A. FAthi - M. Zavidovique, Insertion of $C^{1,1}$ functions and Ilmanen's Lemma.

[4] A. Fathi - A. Siconolfi, Existence of $C^{1}$ critical subsolutions of the Hamilton-Jacobi equation. Invent. Math., 155, no. 2 (2004), pp. 363-388.

[5] T. Ilmanen, The Level-Set Flow on a Manifold, Proc. Symposia Pure Math., 54, no. 1 (1993), pp. 193-204.

[6] J. M. LaSRY - P. L. Lions, A Remark on Regularization in Hilbert Spaces, Israel Math. J., 55, no. 3 (1996), pp. 257-266.

[7] M. Zavidovique, Existence of $C^{1,1}$ critical subsolutions in discrete weak $K A M$ theory. To appear in Journal of Modern Dynamics.

Manoscritto pervenuto in redazione il 6 luglio 2010. 